\title{
Erratum to Machine Learning Prediction of Cyanobacterial Toxin (Microcystin) Toxicodynamics in Humans
}

Stefan Altanerl\#, Sabrina Jaeger2\#, Regina Fotler1, Ivan Zemskov³, Valentin Wittmann³, Falk Schreiber2,4 and Daniel R. Dietrich ${ }^{1}$

${ }^{1}$ Human and Environmental Toxicology, University of Konstanz, Konstanz, Germany; ${ }^{2}$ Life Science Informatics, University of Konstanz, Konstanz, Germany; ${ }^{3}$ Organic and Bioorganic Chemistry, University of Konstanz, Konstanz, Germany; ${ }^{4}$ Faculty of IT, Monash University, Melbourne, Australia \# contributed equally

In this manuscript, which appeared in ALTEX (2020), 37(1), 24-36, doi:10.14573/altex.1904031, there were errors in Tables 1 and 3.

Tab. 1: IC ${ }_{50}$ values of the tested MC congeners on rPPP1, hPPP2A and hPPP5

$\mathrm{IC}_{50}$ were calculated after 5PL-nonlinear regression of at least 3 (hPPP2A) or 5 (rPPP1 and hPPP5) individual replicates using technical duplicates or triplicates; n.d. not determined (PPP2A no longer available, discontinued by manufacturer).

\begin{tabular}{|c|c|c|c|c|c|c|c|c|c|}
\hline \multirow[b]{2}{*}{ Congener } & \multicolumn{3}{|l|}{ rPPP1 } & \multicolumn{3}{|c|}{ hPPP2A } & \multicolumn{3}{|l|}{ hPPP5 } \\
\hline & $\begin{array}{l}\mathrm{IC}_{50} \\
(\mathrm{nM})\end{array}$ & $\mathrm{Cl}_{95}(\mathrm{nM})$ & $\mathbf{R}^{2}$ & $\begin{array}{l}I_{50} \\
(n M)\end{array}$ & $\mathrm{Cl}_{95}(\mathrm{nM})$ & $\mathbf{R}^{2}$ & $\begin{array}{l}I_{50} \\
(n M)\end{array}$ & $\mathrm{Cl}_{95}(\mathrm{nM})$ & $\mathbf{R}^{2}$ \\
\hline MC-RR & 1.5 & $1.3-1.8$ & 0.95 & 1.6 & $1.4-1.7$ & 0.99 & 11.7 & $8.3-16.5$ & 0.96 \\
\hline MC-LR & 0.3 & $0.2-0.4$ & 0.93 & 0.5 & $0.4-0.5$ & 0.99 & 5.1 & $4.0-6.6$ & 0.97 \\
\hline MC-WR & 1.3 & $1.2-1.5$ & 0.99 & n.d. & n.d. & n.d. & 5.1 & $4.3-6.1$ & 0.99 \\
\hline MC-YR & 1.2 & $1.0-1.5$ & 0.94 & 1.0 & $0.8-1.1$ & 0.97 & 5.6 & $4.2-7.6$ & 0.97 \\
\hline MC-LW & 1.9 & $1.4-2.7$ & 0.86 & 0.7 & $0.5-0-9$ & 0.93 & 6.1 & $4.3-8.7$ & 0.96 \\
\hline MC-LY & 0.8 & $0.7-0.9$ & 0.99 & n.d. & n.d. & n.d. & 4.1 & $3.1-5.4$ & 0.97 \\
\hline MC-LA & 2.0 & $1.5-2.6$ & 0.90 & 1.4 & $1.3-1.4$ & 0.99 & 4.7 & $3.5-6.3$ & 0.97 \\
\hline MC-LF & 1.2 & $1.0-1.4$ & 0.97 & 0.7 & $0.7-0.8$ & 0.99 & 2.5 & $2.0-3.2$ & 0.98 \\
\hline MC-HilR & 0.6 & $0.5-0.8$ & 0.99 & n.d. & n.d. & n.d. & 4.2 & $3.5-5.1$ & 0.99 \\
\hline MC-HtyR & 0.7 & $0.6-0.8$ & 0.99 & n.d. & n.d. & n.d. & 4.7 & $3.6-6.0$ & 0.96 \\
\hline (Asp3)MC-RR & 45.0 & $39.3-51.6$ & 0.99 & n.d. & n.d. & n.d. & 167.1 & $131.8-211.8$ & 0.97 \\
\hline (Asp3)MC-LR & 0.9 & $0.7-1.0$ & 0.99 & n.d. & n.d. & n.d. & 10.2 & $8.3-12.5$ & 0.99 \\
\hline (Asp3,Dhb7)MC-RR & 62.0 & $51.7-74.3$ & 0.96 & 84.3 & $80.7-87.8$ & 0.99 & 877.1 & $692.6-1111$ & 0.97 \\
\hline MC-LY(Prg) & 1.7 & $1.3-2.2$ & 0.95 & 0.4 & $0.2-0.3$ & 0.99 & 1.7 & $1.2-2.6$ & 0.95 \\
\hline (DhaSePhe7)MC-LY(Prg) & 1.9 & $1.6-2.4$ & 0.97 & 0.9 & $0.7-1.1$ & 0.94 & 18.2 & $10.7-31.1$ & 0.91 \\
\hline (Enantio-Adda5)MC-LF & - & - & - & - & - & - & - & - & - \\
\hline (Amba5)MC-LY(Prg) & 520,817 & $\begin{array}{l}449,800- \\
603,048\end{array}$ & 0.98 & 2,135 & $\begin{array}{l}1,991 \text { - } \\
2,291\end{array}$ & 0.99 & 54,063 & $\begin{array}{l}37,431 \text { - } \\
78,087\end{array}$ & 0.95 \\
\hline (Anda5)MC-LY(Prg) & 1,724 & $\begin{array}{l}1,434- \\
2,072\end{array}$ & 0.98 & n.d. & n.d. & n.d. & 2,420 & $\begin{array}{l}1,690- \\
3,467\end{array}$ & 0.96 \\
\hline
\end{tabular}

1 doi:10.14573/altex.1904031e 
Tab. 3: MC congener toxicity equivalency factors (TEF)

\begin{tabular}{|c|c|c|c|c|c|c|}
\hline \multirow[t]{2}{*}{ Congener } & \multicolumn{2}{|l|}{ PPP1 } & \multicolumn{2}{|l|}{ PPP2A } & \multicolumn{2}{|l|}{ PPP5 } \\
\hline & $\mathrm{IC}_{50}(\mathrm{nM})$ & TEF & $\mathrm{IC}_{50}(\mathrm{nM})$ & TEF & $\mathrm{IC}_{50}(\mathrm{nM})$ & TEF \\
\hline MC-RR & 1.48 & 0.20 & 1.55 & 0.31 & 11.72 & 0.44 \\
\hline MC-LR & 0.29 & 1.00 & 0.48 & 1.00 & 5.10 & 1.00 \\
\hline MC-WR & 1.33 & 0.22 & n.d. & n.d. & 5.09 & 1.00 \\
\hline MC-YR & 1.23 & 0.24 & 0.97 & 0.50 & 5.60 & 0.91 \\
\hline MC-LW & 1.95 & 0.15 & 0.67 & 0.71 & 6.07 & 0.84 \\
\hline MC-LY & 0.80 & 0.36 & n.d. & n.d. & 4.12 & 1.24 \\
\hline MC-LA & 2.00 & 0.15 & 1.35 & 0.36 & 4.69 & 1.09 \\
\hline MC-LF & 1.19 & 0.24 & 0.73 & 0.66 & 2.53 & 2.02 \\
\hline MC-HilR & 0.60 & 0.49 & n.d. & n.d. & 4.24 & 1.20 \\
\hline MC-HtyR & 0.65 & 0.45 & n.d. & n.d. & 4.67 & 1.09 \\
\hline (Asp3)MC-RR & 45.03 & 0.01 & n.d. & n.d. & 167.10 & 0.03 \\
\hline (Asp3)MC-LR & 0.85 & 0.34 & n.d. & n.d. & 10.19 & 0.50 \\
\hline (Asp3,Dhb7)MC-RR & 61.95 & 0.01 & 84.16 & 0.01 & 877.10 & 0.01 \\
\hline MC-LY(Prg) & 1.69 & 0.17 & 0.27 & 1.76 & 1.73 & 2.95 \\
\hline (DhaSePhe7)MC-LY(Prg) & 1.94 & 0.15 & 0.89 & 0.54 & 18.23 & 0.28 \\
\hline Enantio-LF & - & - & - & - & - & - \\
\hline Amba & 520,817 & 0.000 & 2,135 & 0.000 & 54,063 & 0.00 \\
\hline Anda & 1,724 & 0.000 & n.d. & n.d. & 2,420 & 0.00 \\
\hline
\end{tabular}

n.d., not determined 